\title{
Impact of Diuron contamination on blood cockles (Tegillarca granosa Linnaeus,1758)
}

\begin{abstract}
Examination of the impact of Diuron contamination on blood cockles (Tegillarca granosa) was conducted by combining field screening at three sampling events and a toxicity test. Diuron was extracted using the liquid-liquid extraction (LLE) technique and analyzed using HPLCUV. The median lethal concentration (LC50) of Diuron on T. granosa was tested under a 72-h exposure. Diuron in water samples ranged from not detected (ND) to $3910 \mathrm{ppb}$, which was the highest concentration detected in samples after the irrigation water was discharged from the paddy plantation. Diuron was not detected in sediment samples. Mortality of T. granosa ranged from 4.74 to $38.33 \%$ with the highest percentages recorded after the release of the irrigation water. The LC50 value of Diuron was $1.84 \mathrm{ppm}$. This study suggests that irrigation water from paddy plantation that drifts to coastal areas containing Diuron harms T. granosa at the study area.
\end{abstract}

Keyword: Agriculture; Biocide; Pollution; Acute; Marine; Bivalve 